\title{
Indicadores antropométricos como marcadores de risco para anormalidades metabólicas
}

\author{
Anthropometric indicators \\ as risk markers for metabolic abnormalities
}

João Felipe Mota ${ }^{1}$

Ana Elisa Madalena Rinaldi ${ }^{1}$

Avany Fernandes Pereira ${ }^{2}$

Fábio Lera Orsatti ${ }^{1}$

Roberto Carlos Burini ${ }^{1}$

${ }^{1}$ Centro de Metabolismo em Exercício e Nutrição (CeMENutri), Departamento de Saúde Pública, Faculdade de Medicina de Botucatu, Universidade Estadual Paulista (UNESP). Distrito de Rubião Júnior s/n. 18618-970 Botucatu SP jfemota@yahoo.com.br

${ }^{2}$ Instituto de Nutrição, Universidade Federal do Rio de Janeiro (UFRJ).
Abstract The purpose of this study was to determine which anthropometric indicator has the greatest bearing on the metabolic abnormalities in participants of a Lifestyle Change Program. It consisted of an exploratory, transversal and analytical survey, which assessed the body mass index (BMI), waist circumference (WC), percentage of body fat (\%BF) and of muscle mass (\%MM) of 273 adults and elderly subjects (over 40 years of age). Blood samples after an 8-hour diet were obtained to assess total cholesterol, high-density cholesterol, low-density cholesterol, triacylglycerol and glucose. Statistical analyses for differentiation between the groups and determination of associations were conducted. The level of significance was set at $p<0.05$. When the metabolic abnormalities were assessed as a dependent variable and $B M I, W C, \% B F, \% M M$ as independent variables, it was seen that WC was the anthropometric indicator that showed the closest association with all metabolic abnormalities $(P<0,0001)$, followed by \%MM. The conclusion reached was that WC rather than BMI was the main marker of anthropometric risk for metabolic abnormalities frequently related to obesity. Given the same WC value, overweight and obese individuals had comparable health risks to eutrophic individuals.

Key words Body composition, Obesity, Waist circumference, Body mass index
Resumo O objetivo do presente trabalho foi avaliar qual indicador antropométrico apresenta maior relação com as anormalidades metabólicas em participantes de um programa de Mudança de Estilo de Vida. Tratou-se de uma pesquisa do tipo exploratória, transversal e analítica, na qual foram avaliados 273 adultos e idosos (idade superior a 40 anos) quanto ao Indice de Massa Corporal (IMC), circunferência cintura (CC), \% gordura corporal (GT) e \% massa muscular (\%MM). Foi colhida amostra de sangue em jejum para dosagem de colesterol total e frações, triacilglicerol e glicose. Foram realizadas análises estatísticas para diferenciação entre os grupos e determinação de associações. O nível de significância adotado foi de $p<0,05$. Ao avaliar as anormalidades metabólicas como variável dependente e IMC, CC, GT, \%MM como variáveis independentes, observamos que a CC foi o indicador antropométrico que mostrou melhor associação com todas as anormalidades metabólicas $(p<0,0001)$, seguida da \%MM. Concluise que as anormalidades metabólicas comumente associadas à obesidade apresentam como principal marcador de risco antropométrico a CC e não o IMC. Dado um mesmo valor de CC, sobrepesos e obesos apresentaram riscos à saúde comparáveis aos indivíduos eutróficos.

Palavras-chave Composição corporal, Obesidade, Circunferência da cintura, Indice de massa corporal 


\section{Introdução}

A avaliação da composição corporal é fundamental visto que permite conhecer o estado nutricional de populações e embasar as condutas dietéticas adequadas. Além disso, observa-se relação significativa da gordura corporal com anormalidades metabólicas e risco aumentado para desenvolvimento de doenças crônicas não transmissíveis (DCNTs) ${ }^{1}$. A escolha do método a ser utilizado dependerá de sua validade, aplicabilidade, custo, disponibilidade e também do compartimento corporal a ser mensurado ${ }^{2}$.

As principais anormalidades corporais relacionadas ao desenvolvimento das DCNTs são o excesso de peso corporal por aumento de gordura e a redução de massa muscular ${ }^{3,4}$. A incidência destas doenças pode ser até quatro vezes maior entre homens e mulheres obesos, respectivamente, quando comparados à população não obesa ${ }^{5}$.

O Îndice de Massa Corpórea (IMC) é um dos indicadores antropométricos mais utilizados por ser seguro, de fácil aplicação e ter baixo custo. Além disso, o IMC é reconhecido como preditor de morbidades e mortalidade de diversas DCNTs ${ }^{6}$. Entretanto, este índice apresenta como desvantagem a não identificação do compartimento corporal alterado e sua localização exata ${ }^{7}$.

Outro indicador de fácil aplicabilidade e custo reduzido é a medida da circunferência da cintura (CC). Sua aplicação na prática clínica e em estudos é justificada por constituir principal fator de risco para doenças cardiovasculares. Esta associação pode ser explicada pelo acúmulo de tecido adiposo na região abdominal, promovendo resistência insulínica, dislipidemia e hipertensão arterial sistêmica ${ }^{8}$.

Scarsella e Després ${ }^{9}$, em revisão bibliográfica, afirmam que a adiposidade abdominal está associada com anormalidades metabólicas. A associação entre a circunferência da cintura elevada e a hipertrigliceridemia pode identificar portadores da tríade metabólica aterogênica (concentrações elevadas de insulina, apolipoproteína B e LDL-colesterol pequenas e densas), responsável por aumentar o risco de doença arterial coronariana em 20 vezes.

A elevada porcentagem de gordura corporal total (GT) também está relacionada ao aumento da mortalidade. Zhu et al. ${ }^{10}$ mostraram que a GT estabelece risco para o desenvolvimento da síndrome metabólica.

Outro compartimento corporal importante é o tecido muscular. Este desenvolve papel central no metabolismo proteico, servindo como principal reservatório de aminoácidos, compostos precursores da gliconeogênese hepática, sustentação do corpo e oxidação dos substratos energéticos. Atualmente, a redução da massa muscular (MM), denominada sarcopenia, pode aumentar o risco para DCNTs e também está relacionada com a inabilidade física e a osteoporose ${ }^{4}$.

Deste modo, o presente estudo foi conduzido para avaliar qual indicador antropométrico, dentre os utilizados rotineiramente na prática clínica para avaliação nutricional de adultos e idosos, apresenta maior relação com as anormalidades metabólicas em indivíduos adultos participantes de um programa de mudança de estilo de vida.

\section{Métodos}

\section{População de estudo}

O presente estudo foi realizado no período de fevereiro de 2006 até junho de 2007. Tratou-se de um estudo transversal analítico do qual fizeram parte 273 indivíduos de ambos os sexos, com idade superior a 40 anos, participantes do projeto de extensão universitária "Mexa-se PróSaúde" do município de Botucatu, SP, atendidos por demanda espontânea. Os critérios de exclusão do estudo foram o uso de fármacos hipoglicemiantes e/ou hipolipemiantes $(\mathrm{n}=28)$ e diagnóstico clínico de doenças crônicas que interferissem significativamente na composição corporal, como insuficiência renal, hepática, respiratória, neoplasias e síndrome da imunodeficiência adquirida.

O estudo seguiu os padrões exigidos pela Declaração de Helsinki e foi aprovado pelo Comitê de Ética em Pesquisa da Faculdade de Medicina de Botucatu-SP. Todos os indivíduos assinaram o termo de consentimento livre e esclarecido conforme resolução no 196/96 sobre Pesquisas envolvendo seres humanos, do Conselho de Saúde do Ministério da Saúde, informando-os sobre a proposta e os procedimentos do estudo.

\section{Avaliação dos indicadores antropométricos}

Foram utilizados como indicadores antropométricos o IMC, CC, GT e \%MM. Foram aferidos o peso corporal $(\mathrm{kg})$ e a estatura $(\mathrm{m})$ para posterior cálculo do IMC. Para a aferição do peso, os indivíduos ficaram descalços e vestidos com o mínimo possível de roupas, conforme recomendado por Heyward e Stolarczyk ${ }^{11}$ A balança utilizada foi tipo plataforma digital (Filizola ${ }^{\circledR}$, Bra- 
sil), com capacidade de $150 \mathrm{~kg}$ e escala de divisões de $100 \mathrm{~g}$. A estatura foi avaliada por estadiômetro fixo na parede, devendo o indivíduo estar descalço, com os braços ao longo do corpo ereto, e com 5 pontos encostados na parede: calcanhares, panturrilha, quadril, ombros e cabeça, respeitando-se o plano horizontal de Frankfurt: mantendo os olhos fixos em um plano horizontal, paralelo ao chão. A medição foi feita em duplicata, com a finalidade de se obter a média dos valores, sendo que a diferença entre eles não deveria ultrapassar $1,5 \mathrm{~mm}^{11}$. O diagnóstico de sobrepeso e obesidade, pelo IMC, foi estabelecido quando os indivíduos apresentaram valores entre 25 e $29,9 \mathrm{~kg} / \mathrm{m}^{2}$ e valores iguais e superiores a $30 \mathrm{~kg} / \mathrm{m}^{2}$, respectivamente ${ }^{6}$.

A CC foi medida com fita métrica inextensível e inelástica no ponto médio entre a última costela e a crista ilíaca anterior ${ }^{11}$. Para realização desta medida, o avaliador deve prestar atenção se a fita não está retorcida, a localização correta, exercendo leve pressão, sendo somente necessária uma tomada de medida. De acordo com outros estudos, a localização desta medida representa a região entre a quarta e a quinta vértebra lombar, que em estudos com ressonância magnética em multicortes mostraram melhor associação com a síndrome metabólica e o risco coronariano ${ }^{12,13}$. As medidas foram analisadas de acordo com o sexo, sendo masculino alterada maior ou igual a $94 \mathrm{~cm}$ e muito alterada maior ou igual a $102 \mathrm{~cm}$; mulheres alterada maior ou igual a $80 \mathrm{~cm}$ e muito alterada maior ou igual a $88 \mathrm{~cm}^{6}$.

A GT e a \%MM foram aferidas por impedância bioelétrica (impedancemeter Quantum BIA-101Q, Clinton Township), utilizando para os cálculos a equação proposta por Segal et al. ${ }^{14} \mathrm{e}$ Janssen et al. ${ }^{15}$, respectivamente. Para a realização do teste, os indivíduos permaneceram em jejum de 12 horas, normalmente hidratados (ingestão de 1,5 a 2 litros de água no dia anterior), sem o uso de medicamentos e substâncias diuréticas (álcool ou produtos cafeínados) e sem prática de exercícios físicos nas 24 horas anteriores ao exame. O teste foi realizado no lado dominante do indivíduo, na posição deitada e supina com os braços abertos, em ângulo de $30^{\circ} \mathrm{em}$ relação ao seu corpo.

A classificação da GT foi analisada de acordo com a faixa etária e sexo dos indivíduos ${ }^{16}$, sendo para mulheres e homens, respectivamente, com idade entre 40 a 59 anos: desnutrição $\leq 23 \%$ e $\leq$ $11 \%$; eutrofia: $24-33 \%$ e $12-21 \%$; sobrepeso: $34-$
$39 \%$ e $22-27 \%$; obesidade $\geq 40 \%$ e $\geq 28 \%$; com idade entre 60 a 65 anos: desnutrição $\leq 24 \%$ e $\leq$ $12 \%$; eutrofia: $25-35 \%$ e $14-24 \%$; sobrepeso: $36-$ $41 \%$ e $25-29 \%$; obesidade $\geq 42 \%$ e $\geq 30 \%$. Para a $\% \mathrm{MM}$ foi estabelecido que valores menores ou iguais a $37 \%$ para homens e $28 \%$ para mulheres caracterizava a sarcopenia ${ }^{3}$.

\section{Avaliação bioquímica}

Para análise das variáveis bioquímicas foi colhido sangue venoso após jejum de 12 horas. As avaliações do colesterol total, HDL-colesterol, triacilglicerol e glicose foram realizadas pelo método enzimático colorimétrico em analisador automático System Vitros Chemistry 950 Xrl (Johnson \& Johnson). As concentrações de LDL-colesterol foram calculadas pela fórmula de Friedewald et al. e o não HDL colesterol (nHDL) pelo colesterol total menos HDL-c ${ }^{17}$. Foram consideradas como anormalidades metabólicas: glicose de jejum maior igual a $100 \mathrm{mg} / \mathrm{dL}^{18}$, colesterol total maior igual a $200 \mathrm{mg} / \mathrm{dL}^{19}$, HDL-c menor igual a $40 \mathrm{mg} / \mathrm{dL}$ para homens e menor igual a $50 \mathrm{mg} / \mathrm{dL}$ para mulheres ${ }^{19}$, triacilglicerol maior igual a $150 \mathrm{mg} / \mathrm{dL}^{19}$, LDL-c maior igual a $130 \mathrm{mg} / \mathrm{dL}^{19} \mathrm{e}$ nHDL maior igual ao percentil $75(192 \mathrm{mg} / \mathrm{dL})$.

\section{Análise estatística}

Foi analisada a distribuição das variáveis por meio dos testes Shapiro-Wilk e Kolmogorov \& Smirnov, e a análise de homocedasticidade das variâncias por meio do teste de Levene. Os resultados foram expressos sob a forma de média \pm desvio padrão, acrescido de mediana \pm semiamplitude interquartílica quando a variável não apresentava distribuição normal. Para determinação da associação entre os indicadores antropométricos foram realizadas correlações de Pearson. Para comparação das variáveis numéricas com distribuição normal foi realizado o teste $t$ de Student e ANOVA/MANOVA (pos hoc Duncan's test) e, para as variáveis numéricas com distribuição anormal, os testes de Mann-Whitney e Kruskal-Wallis ANOVA. Foi realizada regressão múltipla em stepwise com as anormalidades metabólicas como variável dependente, e, como independentes, os indicadores antropométricos. O nível de significância adotado foi de $\mathrm{p}<$ 0,05 . Todas as análises foram realizadas utilizando o software STATISTICA for Windows (version 5.1, Statsoft, Tulsa, USA). 


\section{Resultados}

Os indivíduos apresentaram média de idade de $53 \pm 5$ anos, sendo a maior parte representada pelo sexo feminino $(75,5 \%)$. O percentual de indivíduos eutróficos e obesos foi $28,2 \%$ e $31,1 \%$, respectivamente, sendo a maioria dos indivíduos $(40,7 \%)$ diagnosticados como sobrepesos. Na caracterização geral e por sexo, a amostra foi classificada como sobrepeso. Os homens apresentaram maior CC quando comparado às mulheres $(\mathrm{P}<0,001)$, mas a medida em valores médios foi considerada alterada, enquanto que para as mulheres foi muito alterada. Os valores de GT e HDLc foram menores nos homens e de \%MM maiores (Tabela 1).

Após o cálculo do IMC, os indivíduos com diagnóstico de sobrepeso e obesidade apresenta- ram maiores concentrações de glicose $(p<0,05)$, triacilglicerol $(p<0,001), \mathrm{nHDL}$ colesterol $(\mathrm{p}<0,01)$ e menores concentrações de HDL-c $(\mathrm{p}<0,001)$, quando comparados com os eutróficos. Já as concentrações de LDL-c foram superiores $(\mathrm{p}<0,05)$ nos obesos em relação aos eutróficos (Tabela 2).

Os indivíduos que apresentaram a medida da circunferência da cintura alterada ou muito alterada apresentaram concentrações de glicose jejum $(\mathrm{p}<0,05)$, triacilglicerol $(\mathrm{p}<0,0001)$, colesterol total $(\mathrm{p}<0,05)$, nHDL colesterol $(\mathrm{p}<0,001)$, LDL-c $(p<0,05)$ maiores do que aqueles classificados com circunferência normal, e as de HDL-c menores $(\mathrm{p}<0,001)$. Além disso, os indivíduos do estudo com circunferência da cintura muito alterada apresentaram concentrações de glicose em jejum superiores aos demais indivíduos $(\mathrm{p}<0,05)$ (Tabela 3$)$.

Tabela 1. Caracterização geral e por sexo da amostra.

\begin{tabular}{|c|c|c|c|}
\hline & $Q+\sigma^{\prime \prime} \quad(\mathbf{n}=273)$ & $\uparrow \quad(n=206)$ & $O^{7} \quad(\mathbf{n}=67)$ \\
\hline \multicolumn{4}{|l|}{ Características descritivas } \\
\hline Idade (anos) & $53 \pm 10$ & $53 \pm 10$ & $53 \pm 10$ \\
\hline $\operatorname{IMC}\left(\mathrm{kg} / \mathrm{m}^{2}\right)$ & $28,1 \pm 5,0$ & $27,9 \pm 5,1$ & $28,6 \pm 4,7$ \\
\hline Circunferência da cintura $(\mathrm{cm})$ & $94,4 \pm 13,2$ & $88,8 \pm 12,4$ & $99,4 \pm 12,5^{* *}$ \\
\hline$\%$ Gordura total & $31,4 \pm 8,5$ & $33,5 \pm 8,2$ & $24,9 \pm 6,0^{* *}$ \\
\hline$\%$ Massa muscular & $30,6 \pm 5,7$ & $28,3 \pm 3,9$ & $37,8 \pm 4,6^{* *}$ \\
\hline \multicolumn{4}{|l|}{ Variáveis metabólicas } \\
\hline Glicose jejum $(\mathrm{mg} / \mathrm{dL})^{*}$ & $91,0 \quad 6,0$ & $89,5 \pm 6,5$ & $93,0 \pm 6,6$ \\
\hline Triacilglicerol $(\mathrm{mg} / \mathrm{dL}) *$ & $117,0 \pm 41,0$ & $115,5 \pm 37,0$ & $128,0 \pm 51,5$ \\
\hline Colesterol total $(\mathrm{mg} / \mathrm{dL})$ & $215,4 \pm 37,6$ & $217,1 \pm 36,9$ & $210,1 \pm 39,3$ \\
\hline HDL-c $(\mathrm{mg} / \mathrm{dL})$ & $50,1 \pm 12,8$ & $51,9 \pm 12,9$ & $44,8 \pm 10,6^{* *}$ \\
\hline nHDL colesterol (mg/dL) & $165,3 \pm 38,5$ & $165,2 \pm 38,5$ & $165,3 \pm 38,9$ \\
\hline LDL-c $(\mathrm{mg} / \mathrm{dL})$ & $137,9 \pm 34$ & $138,9 \pm 34,1$ & $135,0 \pm 34,1$ \\
\hline
\end{tabular}

Tabela 2. Descrição das variáveis bioquímicas segundo a classificação do Índice de Massa Corporal de indivíduos adultos e idosos participantes de um projeto de extensão universitária, Botucatu, 2006-2007.

\begin{tabular}{lccr}
\hline \multicolumn{1}{c}{ Variáveis } & Eutrófico $(\mathbf{n}=\mathbf{7 7})$ & Sobrepesos $(\mathbf{n}=\mathbf{1 1 1})$ & Obesos $(\mathbf{n}=\mathbf{8 5})$ \\
\hline Glicemia $(\mathrm{mg} / \mathrm{dL})^{*}$ & $89 \pm 12,0$ & $92 \pm 11,0^{* *}$ & $91 \pm 16,0^{* *}$ \\
Triacilglicerol $(\mathrm{mg} / \mathrm{dL})^{*}$ & $92 \pm 57,0$ & $119 \pm 98^{* *}$ & $134 \pm 84^{* *}$ \\
HDL-c $(\mathrm{mg} / \mathrm{dL})$ & $55,5 \pm 12,4$ & $47,6 \pm 12,1^{* *}$ & $48,6 \pm 12,8^{* *}$ \\
Colesterol total (mg/dL) & $209,4 \pm 34,9$ & $217,5 \pm 39,5$ & $218,1 \pm 37,1$ \\
nHDL $(\mathrm{mg} / \mathrm{dL})$ & $153,9 \pm 35,3$ & $169,9 \pm 40,5^{* *}$ & $169,5 \pm 37^{* *}$ \\
LDL-c $(\mathrm{mg} / \mathrm{dL})$ & $131,6 \pm 32,9$ & $141,3 \pm 35,2$ & $139,3 \pm 33,1^{* *}$ \\
\hline
\end{tabular}

Valores descritos em média \pm desvio padrão. "Mediana \pm semi-amplitude interquartílica. nHDL: não HDL colesterol (colesterol total-HDL-c). " $\mathrm{p}<0,05$ diferenças em relação aos eutróficos. 
Quando realizada a classificação pela porcentagem de gordura corpórea total, os resultados apresentaram-se controversos, sendo que as concentrações de HDL-c foram menores apenas nos indivíduos com sobrepeso quando comparados aos eutróficos $(\mathrm{p}<0,02)$ e as de triacilglicerol superiores nos obesos quando comparados aos eutróficos $(\mathrm{p}<0,001)$ (Tabela 4).

Com relação à massa muscular, os indivíduos com diagnóstico de sarcopenia tiveram concentrações superiores de glicose em jejum $(p<0,05)$, triacilglicerol $(p<0,001)$, nHDL colesterol $(\mathrm{p}<0,01)$, colesterol total $(\mathrm{p}<0,05)$ e tendência a apresentarem concentrações menores de HDL-c $(\mathrm{p}<0,09)$ (Tabela 5).

No modelo de regressão múltipla em stepwi$s e$, avaliando as anormalidades metabólicas como variável dependente e IMC, CC, GT, \%MM como variáveis independentes, observamos que a circunferência da cintura foi a variável independente significativa $(p<0,001)$, determinante da maioria das anormalidades metabólicas, seguida da \%MM (Tabela 6). A GT apresentou associação com algumas desordens, porém sem significância. Já o IMC apresentou associação apenas com a hipertrigliceridemia, todavia, de maneira inversa, ou seja, quanto maior o IMC, menor a concentração de triacilglicerol (Tabela 6).

\section{Discussão}

A avaliação do estado nutricional é de extrema importância, pois é capaz de diagnosticar anormalidades corporais, permitindo assim avaliar o grau de risco para o desenvolvimento de DCNTs. Desse modo, o uso dos indicadores antropométricos é uma ferramenta indispensável nesta avaliação e a escolha pelo indicador mais representativo de desordens metabólicas é fundamental.

Foi encontrada elevada prevalência $(71,8 \%)$ de indivíduos com excesso de peso corporal. Dados do Instituto Brasileiro de Geografia e Estatística ${ }^{20}$ mostram que em 2003 foram diagnosticados como portadores de excesso de peso corporal cerca de $40 \%$ dos indivíduos com idade

Tabela 3. Descrição das variáveis bioquímicas segundo a classificação da circunferência da cintura de indivíduos adultos e idosos participantes de um projeto de extensão universitária, Botucatu, 2006-2007.

\begin{tabular}{lccc}
\hline \multicolumn{1}{c}{ Variáveis } & Normal $(\mathbf{n}=\mathbf{6 7})$ & Alterada $(\mathbf{n}=\mathbf{7 9})$ & Muito alterada $(\mathbf{n}=\mathbf{1 2 7})$ \\
\hline Glicemia $(\mathrm{mg} / \mathrm{dL})^{*}$ & $89 \pm 11,0$ & $89 \pm 10,0$ & $94 \pm 15,0^{* * * * *}$ \\
Triacilglicerol (mg/dL) & $83 \pm 44$ & $127 \pm 104^{* *}$ & $131 \pm 90^{* *}$ \\
HDL-c $(\mathrm{mg} / \mathrm{dL})$ & $55,7 \pm 12,4$ & $48,5 \pm 13,0^{* *}$ & $48,2 \pm 12,1^{* *}$ \\
Colesterol total (mg/dL) & $205,4 \pm 36$ & $218,6 \pm 38,8^{* *}$ & $218,7 \pm 36,9^{* *}$ \\
nHDL (mg/dL) & $149,7 \pm 34,7$ & $170,1 \pm 38,8^{* *}$ & $170,5 \pm 38,4^{* *}$ \\
LDL-c (mg/dL) & $130,8 \pm 33,9$ & $141,2 \pm 34^{* *}$ & $139,6 \pm 33,9^{* *}$ \\
\hline
\end{tabular}

Valores descritos em média \pm desvio padrão. *Mediana \pm semi-amplitude interquartílica. nHDL: não HDL colesterol (colesterol total-HDL-c). ${ }^{* *}<<0,05$ em relação ao grupo normal. ${ }^{* * *} \mathrm{p}<0,05$ entre o grupo muito alterada e alterada.

Tabela 4. Descrição das variáveis bioquímicas segundo a classificação da porcentagem de gordura corpórea total de indivíduos adultos e idosos participantes de um projeto de extensão universitária, Botucatu, 2006-2007.

\begin{tabular}{lccc}
\hline \multicolumn{1}{c}{ Variáveis } & Eutrófico $(\mathbf{n = 1 7 2})$ & Sobrepesos $(\mathbf{n = 1 3})$ & Obesos $(\mathbf{n}=\mathbf{8 4})$ \\
\hline Glicemia $(\mathrm{mg} / \mathrm{dL})^{*}$ & $90 \pm 11,0$ & $94 \pm 9,0$ & $91,5 \pm 16,0$ \\
Triacilglicerol $(\mathrm{mg} / \mathrm{dL})^{*}$ & $109,5 \pm 69,5$ & $96 \pm 161$ & $139,5 \pm 84,0^{* *}$ \\
HDL-c $(\mathrm{mg} / \mathrm{dL})$ & $51,4 \pm 13,5$ & $43,8 \pm 10,6^{* *}$ & $47,9 \pm 10,9$ \\
Colesterol total (mg/dL) & $215,5 \pm 38,3$ & $209,2 \pm 31,5$ & $217,7 \pm 37,1$ \\
NHDL (mg/dL) & $164,1 \pm 39,2$ & $165,4 \pm 34$ & $169,8 \pm 37,1$ \\
LDL-c $(\mathrm{mg} / \mathrm{dL})$ & $138,2 \pm 34,6$ & $134,4 \pm 30,7$ & $139,5 \pm 33,3$ \\
\hline
\end{tabular}

Valores descritos em média \pm desvio padrão. * Mediana \pm semi-amplitude interquartílica. nHDL: não HDL colesterol (colesterol total-HDL-c).** $\mathrm{p}<0,05$ em relação aos eutróficos. 
superior a 20 anos. Santos e Sichieri ${ }^{21}$, avaliando indivíduos acima de 40 anos de idade, encontraram que cerca de 50\% dos idosos apresentavam obesidade. Além disso, a circunferência da cintura alterada foi superior a 50\% nas mulheres e $40 \%$ nos homens ${ }^{21}$.

No presente estudo foi observado que a circunferência da cintura exerceu maior influência sobre as anormalidades metabólicas estudadas, seguida da \% de massa muscular somente para as anormalidades nas concentrações de HDL-c, colesterol total e nHDL.

A GT foi o indicador que menos obteve relação com as variáveis bioquímicas estudadas. Este resultado não foi decorrente dos valores de referência utilizados, foram testados outros pontos de corte durante o estudo e não foram encontradas diferenças significativas com os mes$\operatorname{mos}^{11,22,23}$. Além disso, os valores utilizados para classificação da GT consideram o sexo e a faixa etária do indivíduo, sendo, assim, são considera-

Tabela 5. Descrição das variáveis bioquímicas segundo a classificação da porcentagem de massa muscular de indivíduos adultos e idosos participantes de um projeto de extensão universitária, Botucatu, 2006-2007.

\begin{tabular}{lrc}
\hline \multicolumn{1}{c}{ Variáveis } & \multicolumn{1}{c}{$\begin{array}{c}\text { Normal } \\
(\mathbf{n}=141)\end{array}$} & $\begin{array}{c}\text { Sarcopenia } \\
(\mathbf{n}=132)\end{array}$ \\
\hline${\text { Glicemia }(\mathrm{mg} / \mathrm{dL})^{*}}_{\text {Triacilglicerol }(\mathrm{mg} / \mathrm{dL})^{*}}$ & $89 \pm 10,0$ & $92 \pm 16,0^{* *}$ \\
HDL-c $(\mathrm{mg} / \mathrm{dL})$ & $51,8 \pm 65,0$ & $129 \pm 95,5^{* *}$ \\
Colesterol total $(\mathrm{mg} / \mathrm{dL})$ & $211,7 \pm 37,4$ & $48,4 \pm 12,2^{* *}$ \\
nHDL $(\mathrm{mg} / \mathrm{dL})$ & $159,9 \pm 37,8$ & $171 \pm 38,7^{* *}$ \\
LDL-c $(\mathrm{mg} / \mathrm{dL})$ & $135,2 \pm 33,2$ & $140,9 \pm 34,8$ \\
\hline
\end{tabular}

Valores descritos em média \pm desvio padrão. *Mediana \pm semiamplitude interquartílica. nHDL: não HDL colesterol (colesterol total-HDL-c). ${ }^{* *} \mathrm{p}<0,05$ em relação ao normal. dos adequados para um bom diagnóstico do estado nutricional ${ }^{16}$.

No modelo de regressão, o IMC não apresentou relação com as anormalidades metabólicas estudadas, sendo observada associação inversa com o TG. Janssen et $\mathrm{al}^{24}$ verificaram que tanto o IMC quanto a CC isolados são fortes fatores preditivos para comorbidades relacionadas à obesidade. Entretanto, quando ambos foram avaliados conjuntamente, somente a CC teve associação positiva e significativa com tais comorbidades. Deste modo, os autores sugerem que a CC é o melhor parâmetro antropométrico, sendo necessária melhor estratificação dos seus pontos de corte. O IMC não reflete qual compartimento corporal está alterado, sendo a adiposidade abdominal responsável pela maior liberação ácidos graxos livres, contribuindo com o aumento da síntese hepática de $\mathrm{TG}^{25}$.

Koning et al. ${ }^{8}$, em meta análise, evidenciaram que a CC pode ser considerada fator de risco para doenças cardiovasculares (DCVs). Além disso, o aumento de um centímetro da CC pode aumentar em $2 \%$ o risco para DCV. Os autores sugerem também que a medida da CC deve ser incluída na rotina para avaliação para risco cardiovascular.

Da Silva et al. ${ }^{26}$ observaram correlação positiva entre CC e pressão arterial, resistência insulínica e triacilglicerois, indicando que não apenas a quantidade de tecido adiposo, mas sim sua distribuição, pode influenciar na resistência insulínica e nas alterações metabólicas promovidas pela hiperinsulinemia. Orsatti et al. $^{27}$, ao avaliarem mulheres na pós-menopausa, verificaram que a CC tem maior sensibilidade para detectar anormalidades metabólicas [hipertrigliceridemia, índice aterogênico plasmático, índice de resistência à insulina (HOMA-IR), HDL-c reduzido], en-

Tabela 6. Análise de regressão logística multivariada para determinar quais os indicadores associados às desordens metabólicas, Botucatu, 2006-2007.

\begin{tabular}{lcccccc}
\hline \multicolumn{1}{c}{ Variáveis } & $\begin{array}{c}\mathbf{G l i c o s e}^{\alpha} \beta \\
\left(\mathbf{R}^{2}=\mathbf{0 , 0 4 7 )}\right.\end{array}$ & $\begin{array}{c}\boldsymbol{T} \boldsymbol{G}^{\beta} \beta \\
\left(\mathbf{R}^{2}=\mathbf{0 , 1 3 4 )}\right.\end{array}$ & $\begin{array}{c}\mathbf{H D L}-\mathrm{c}^{\delta} \beta \\
\left(\mathbf{R}^{2}=\mathbf{0 , 1 6 1 )}\right.\end{array}$ & $\begin{array}{c}\mathbf{C T}^{\lambda} \beta \\
\left(\mathbf{R}^{2}=\mathbf{0 , 0 2 9}\right)\end{array}$ & $\begin{array}{c}\mathbf{n H D L} \beta \\
\left(\mathbf{R}^{2}=\mathbf{0 , 0 5 5}\right)\end{array}$ & $\begin{array}{c}\mathbf{L D L}^{\ddagger}-\mathbf{c}^{\dagger} \beta \\
\left(\mathbf{R}^{2}=\mathbf{0 , 0 1 3}\right)\end{array}$ \\
\hline IMC & $-0,20$ & $-0,32^{*}$ & - & - & - & - \\
CC & $0,364^{*}$ & $0,591^{*}$ & $-0,40^{*}$ & - & $0,242^{*}$ & - \\
GT & - & - & - & - & $-0,22$ & - \\
$\% \mathrm{MM}$ & - & - & $-0,20^{*}$ & $-0,17^{*}$ & $-0,20^{*}$ & $-0,12$ \\
\hline
\end{tabular}

IMC: Índice de Massa Corporal; CC: circunferência da cintura; GT: porcentagem de gordura corpórea total; \%MM: porcentagem de massa muscular; TG: triacilglicerois; CT: colesterol total; nHDL: não HDL colesterol. ${ }^{\circledR}$ Glicose $\geq 100 \mathrm{mg} / \mathrm{dL} ;{ }^{\beta} \mathrm{TG} \geq 150 \mathrm{mg} / \mathrm{dL}$; ${ }^{\delta} \mathrm{HDL}-$ $\mathrm{c} \leq 40 \mathrm{mg} / \mathrm{dL}$ para homens ou $\leq 50 \mathrm{mg} / \mathrm{dL}$ para mulheres; ${ }^{\lambda} \mathrm{CT} \geq 200 \mathrm{mg} / \mathrm{dL} ;{ }^{\ddagger} \mathrm{nHDL} \geq 192 \mathrm{mg} / \mathrm{dL}(\mathrm{P} 75) ;{ }^{\dagger} \mathrm{LDL}-\mathrm{c} \geq 130 \mathrm{mg} / \mathrm{dL} .{ }^{*} \mathrm{p}<0,001$. Lacunas não preenchidas indicam ausência de associação. 
quanto o IMC tem maior especificidade. Os autores concluem, assim como o presente estudo, que somente a CC pode indicar risco cardiovascular e metabólico. Em outro estudo, envolvendo adultos e idosos de ambos os sexos, foi observado que a CC foi o indicador antropométrico que melhor se correlacionou com variáveis bioquímicas alteradas (baixas concentrações de HDL-c e altas de triacilglicerois e glicose), bem como com a hipertensão arterial ${ }^{28}$.

Em outro estudo brasileiro foi observado que a correlação entre o IMC e o HOMA-IR foi menor do que entre a CC e o HOMA-IR. Além disso, após o ajuste pelo IMC, a CC manteve correlação com o HOMA-IR, a pressão arterial, a apolipoproteína B e com as concentrações de triacilglicerois $^{29}$.

Onat et al. ${ }^{30}$ estudando indivíduos com síndrome metabólica verificaram que a CC era o melhor preditor de adiposidade visceral, tanto em homens quanto em mulheres. Neste estudo, os autores identificaram que a CC era um fator de risco independente para anormalidades lipídicas, tais como redução da concentração de HDL-c e hipertrigliceridemia.

Em relação à massa muscular, Wolfe $e^{4}$ (2006) discute detalhadamente a importância da manutenção e aumento da massa muscular na preven- ção da obesidade. O ponto central é que quanto maior a massa muscular, maior o turnover proteico, contribuindo para o aumento do gasto energético e da oxidação de gordura. Assim, uma diminuição da massa muscular pode promover redução na capacidade de oxidação da glicose e de ácidos graxos, elevando suas concentrações plasmáticas, estabelecendo um ciclo vicioso pelo aumento dos estresses oxidativo e inflamatório que causam lesão direta no tecido muscular ${ }^{31}$.

Além disso, a sarcopenia, a redução da força e da massa muscular, estão associadas à diminuição do metabolismo basal e consequente desenvolvimento das DCNTs ${ }^{4}$.

A principal limitação do estudo foi o modo de seleção da amostra. Esta foi constituída por demanda espontânea. Pode ser que os participantes deste estudo mostraram interesse pelo programa de mudança de estilo de vida por já estarem com excesso de peso e alguma anormalidade metabólica.

Logo, a circunferência da cintura foi o indicador antropométrico que apresentou maior relação com as anormalidades metabólicas relacionadas à obesidade. Assim, para um mesmo valor de circunferência da cintura, indivíduos com sobrepeso e obesidade apresentavam riscos à saúde comparáveis ao indivíduo eutrófico.

\section{Colaboradores}

JF Mota, AEM Rinaldi, AF Pereira, FL Orsatti e RC Burini participaram igualmente de todas as etapas da elaboração do artigo.

\section{Referências}

1. Rosin BL. The progression of cardiovascular risk to cardiovascular disease. Rev Cardiovasc Med 2007; 8(4): S3-8.

2. Brodie D, Moscrip V, Hutcheon R. Body composition measurement: a review of hydrodensitometry, anthropometry, and impedance methods. Nutrition $1998 ; 14(3): 296-310$.

3. Janssen I, Heymsfield SB, Ross R. Low relative skeletal muscle mass (sarcopenia) in older persons is associated with functional impairment and physical disability. J Am Geriat Soc 2002; 50(5):889-896.

4. Wolfe RR. Underappreciated role of muscle in health and disease. Am J Clin Nutr 2006; 84(3):475-482.

5. Klein S, Sheard NF, Pi-Sunyer X, Daly A, WylieRosett J, Kulkarni K, Clark NG. Weight Management through lifestyle modification for the prevention and management of type 2 diabetes: rationale and strategies. Diabetes Care 2004; 27(8):2067-2073.

6. World Health Organization (WHO), Report of a Joint WHO/FAO Expert Consultation. Diet, nutrition and the prevention of chronic diseases. Expert Consultation on diet, nutrition and prevention of chronic diseases. Geneva (Swissland): WHO/FAO; 2002. 
7. Coppini LZ. Estado Nutricional: Métodos de Avaliação, Diagnóstico e Significado Clínico. In: Magnoni D, Cukier C, organizadores. Perguntas e Respostas em Nutrição Clínica. São Paulo: Roca; 2001. p. 139-146.

8. Koning L, Merchant AT, Pogue J, Anad SS. Waist circumference and waist-to-hip ratio as predictors of cardiovascular events: meta-regression analysis of prospective studies. Eur Heart J 2007; 28(7):850-856.

9. Scarsella C, Després JP. Tratamiento de la obesidad: necesidad de centrar la atención en los pacientes de alto riesgo caracterizados por la obesidad abdominal. Cad Saude Publica 2003; 19(Supl. 1):S7-S19.

10. Zhu S, Wang Z, Shen W, Heymsfield SB, Heshka S. Percentage body fat ranges associated with metabolic syndrome risk: results based on the third $\mathrm{Na}$ tional Health and Nutrition Examination Survey (1988-1994). Am J Clin Nutr 2003; 78(2):228-235.

11. Heyward VH, Stolarczyk LM. Avaliação da composição corporal aplicada. 1 $1^{\text {a }}$ ed. Barueri: São Paulo; 2000.

12. Wong S, Janssen I, Ross R. Abdominal adipose tissue distribution and metabolic risk. Sports Med 2003; 33(10):709-726.

13. Risérus U, Arnlöv J, Brismar K, Zethelius B, Berglund L, Vessby B. Sagittal abdominal diameter is a strong anthropometric marker of insulin resistance and hyperproinsulinemia in obese men. Diabetes Care 2004; 27(8):2041-2046.

14. Segal KR, Van Loan M, Fitzgerald PI, Hodgdon JA, Van Itallie TB. Lean body mass estimation by bioelectrical impedance analysis: a four-site cross-validation study. Am J Clin Nutr 1988; 47(1):7-14.

15. Janssen I, Heymsfield SB, Baumgartner RN, Ross R. Estimation of skeletal muscle mass by bioelectrical impedance analysis. J Appl Physiol 2000; 89(2):465471.

16. Gallagher D, Heymsfield SB, Heo M, Jebb SA, Murgatroyd PR, Sakamoto Y. Healthy percentage body fat ranges: an approach for developing guidelines based on body mass index. Am J Clin Nutr 2000; 72(3):694-701.

17. Frontini MG, Srinivasan SR, Xu JH, Tang R, Bond MG, Berenson G. Utility of non-high-density lipoprotein cholesterol versus other lipoprotein measures in detecting subclinical atherosclerosis in young adults (The Bogalusa Heart Study). Am J Cardiol 2007; 100(1):64-68.

18. American Diabetes Association. Diagnosis and Classification of Diabetes Mellitus. Diabetes Care 2008; 31(Supl. 1):S55-60.

19. Executive Summary of The Third Report Of The National Cholesterol Education Program (NCEP). Expert Panel on the Detection, Evaluation, and Treatment of High Blood Cholesterol in Adults (Adult Treatment Panel III). JAMA 2001; 285(19): 2486-2497.

20. Instituto Brasileiro de Geografia e Estatística (IBGE). Coordenação de Índices de Preços. Pesquisa de Orçamentos Familiares 2002-2003: análise da disponibilidade domiciliar e estado nutricional no Brasil. Rio de Janeiro: IBGE; 2004.
21. Santos DM, Sichieri R. Índice de Massa Corporal e indicadores antropométricos de adiposidade em idosos. Rev Saude Publica 2005; 39(2):163-168.

22. Bray G. An approach to the classification and evaluation of obesity. In: Björntorp P, Brodoff BN. Obesity. Philadelphia: J.B. Lippincott; 1992. p. 294-308.

23. Mcardle WD, Katch FI, Katch VL. Avaliação da composição corporal. In: Mcardle WD, Katch FI, Katch VL, organizadores. Fisiologia do exercício. Rio de Janeiro: Guanabara Koogan; 2003. p. 773-814

24. Janssen I, Baumgartner RN, Ross R, Rosenberg IH, Roubenoff R. Skeletal muscle cutpoints associated with elevated disability risk in older men and women. Am J Epidemiol 2004; 159(4):413-421.

25. Yvon AC, Laurence P, Willy JM. n-3 Fatty acids and the metabolic syndrome. Am J Clin Nutr 2006; 83(Supl. 6):1499S-1504S.

26. Da Silva JL, Barbosa DS, de Oliveira JA, Guedes DP. Distribuição centrípeta da gordura corporal, sobrepeso e aptidão cardiorrespiratória: associação com sensibilidade insulínica e alterações metabólicas. Arq Bras Endocrinol Metabol 2006; 50(6): 1034-1040.

27. Orsatti FL, Nahas EAP, Nahas-Neto J, Maestá N, Padoani NP, Orsatti CL. Indicadores antropométricos e as doenças crônicas não transmissíveis em mulheres na pós-menopausa da região Sudeste do Brasil. Rev Bras Ginecol Obstet 2008; 30(4):182-189.

28. Rezende FAC, Rosado LEFPL, Ribeiro RCL, Vidigal FC, Vasques ACJ, Bonard IS, Carvalho CR. Índice de Massa Corporal e circunferência abdominal: associação com fatores de risco cardiovascular. Arq Bras Cardiol 2006; 87(6):728-734.

29. Da Silva JL, Barbosa DS, de Oliveira JA, Guedes DP. Distribuição centrípeta da gordura corporal, sobrepeso e aptidão cardiorrespiratória: associação com sensibilidade insulínica e alterações metabólicas. Arq Bras Endocrinol Metab 2006; 50(6):1034-1040.

30. Onat A, Avc GS, Barlan MM, Uyarel H, Uzunlar B, Sansoy V. Measures of abdominal obesity assessed for visceral adiposity and relation to coronary risk. Int J Obes Relat Metab Disord 2004; 28(8):1018-1025.

31. Roubenoff R. Catabolism of aging: is it an inflammatory process? Curr Opin Clin Nutr Metab Care 2003; 6(3):295-299.

Artigo apresentado em 03/06/2008

Aprovado em 29/12/2008

Versão final apresentada em 30/01/2009 